\title{
Analytical approach to soliton ratchets in asymmetric potentials
}

\author{
Niurka R. Quintero, ${ }^{1,2, *}$ Bernardo Sánchez-Rey, ${ }^{1, \dagger}$ and Mario Salerno ${ }^{3, \neq}$ \\ ${ }^{1}$ Departamento de Física Aplicada I, E. U. P., Universidad de Sevilla, Virgen de África 7, 41011 Sevilla, Spain \\ ${ }^{2}$ Instituto Carlos I de Física Teórica y Computacional, Universidad de Granada, 18071 Granada, Spain \\ ${ }^{3}$ Dipartimento di Fisica "E.R. Caianiello", Instituto Nazionale di Fisica Nucleare (INFN) \\ and Consorzio Nazionale Interuniversitario per le Scienze Fisiche della Materia (CNISM), Universitá di Salerno, \\ via S. Allende 184081 Baronissi (SA), Italy
}

(Received 4 December 2003; revised manuscript received 21 January 2005; published 13 July 2005)

\begin{abstract}
We use soliton perturbation theory and collective coordinate ansatz to investigate the mechanism of soliton ratchets in a driven and damped asymmetric double sine-Gordon equation. We show that, at the second order of the perturbation scheme, the soliton internal vibrations can couple effectively, in presence of damping, to the motion of the center of mass, giving rise to transport. An analytical expression for the mean velocity of the soliton is derived. The results of our analysis confirm the internal mode mechanism of soliton ratchets proposed in [Phys. Rev. E 65, 025602(R) (2002)].
\end{abstract}

DOI: 10.1103/PhysRevE.72.016610

PACS number(s): 05.45.Yv

\section{INTRODUCTION}

During the past years, a great deal of attention has been devoted to the ratchet effects both for point particles [1-4] and for extended systems [5-8]. Some experimental realizations of these models can be found in [7,9-12] (see [13] for a recent review). Ratchet-like systems such as systems of two particles with an internal degree of freedom [3], periodic rocket ratchets (deterministic and stochastic) [14]; and temperature ratchets [15], have also been considered. For infinite-dimensional systems described by nonlinear partial differential equations (PDEs) of soliton type, ratchet phenomena were investigated both in the case of asymmetric potentials in presence of symmetric forces [16]; and in the case of symmetric potentials with asymmetric forces $[12,17,18]$. In both cases, the ratchet phenomenon manifests as a unidirectional motion of the soliton, similar to the drift motion occurring for point particle ratchets (from here, the name of soliton ratchets). A symmetry approach to the phenomenon, which allows one to establish conditions for the occurrence of soliton ratchets, was developed in Ref. [18]. This approach, although useful for predicting the phenomenon, does not provide information about the actual mechanism responsible for the unidirectional motion. The mechanism underlying soliton ratchets was proposed in [16] for the case of a perturbed asymmetric double sine-Gordon equation (ADSGE) driven by symmetric forces, and extended in Ref. [17] to the case of a perturbed sine-Gordon system in presence of asymmetric drivers. In both cases the phenomenon was ascribed to the existence of an internal oscillations of the kink's width, which, in presence of damping, is able to couple soliton internal vibrations to the translational mode, producing in this way transport. The phenomenon was described as follows: the energy pumped by the ac force into

\footnotetext{
*Electronic address: niurka@euler.us.es

†Electronic address: bernardo@us.es

*Electronic address: salerno@sa.infn.it
}

the soliton internal mode is converted intoa net dc motion by the coupling of the internal vibration with the center of mass induced by the damping. This internal mode mechanism was confirmed by numerical and analytical investigations for the case of the sine-Gordon system with asymmetric periodic forces [17-19] and by direct numerical investigations for the case of soliton ratchets of the asymmetric double sineGordon equation [16]. In the last case, a detailed numerical investigation (see [16]) showed the following facts:

(i) The presence of damping and the asymmetry of the potential are both crucial ingredients for the existence of the net motion of the soliton (in presence of the ac force, but in absence of damping, the asymmetry of the potential does not produce transport). (ii) The effect of the damping is to couple effectively the internal vibrations of the kink to the motion of the center of mass. (iii) For fixed values of the amplitude and frequency of the ac force there is an optimal value of the damping for which the transport is maximal. (iv) The direction of the motion is fixed by the asymmetry of the potential and is independent of initial conditions. (v) For fixed values of the damping, the average velocity of the kink shows a resonant behavior as a function of the frequency of the ac force. (vi) At low damping and higher forcing strengths, current reversals in the kink dynamics can occur. This phenomenon was ascribed in Ref. [16] to the soliton-phonon interaction rather than to the internal mode mechanism.

These points were also found to be valid for different soliton ratchet systems $[17,20]$. In Ref. [20], the existence of an optimal value of the damping constant which maximizes the transport, was ascribed to the relativistic nature of the kink dynamics and the possibility of nonzero currents in absence of damping, was also found.

The aim of this paper is to present an analytical investigation of soliton ratchets of the asymmetric double sineGordon equation that confirms the aforementioned internal mode mechanism proposed in Ref. [16] as well as points (i)-(vi) listed above. To this end, we use perturbation theory and a collective coordinates (CC) for the soliton shape, to derive ordinary differential equations (ODE) for the center of mass and for the soliton width. We show that, to second 
order of perturbation theory, soliton width vibrations couple effectively to the motion of the center of mass via the damping in the system. By using the collective coordinate equations, we are able to derive an analytical expression for the mean drift velocity of the kink as a function of the system parameters. The analysis is shown to be in good agreement with numerical simulation and with the results (i)-(vi) obtained in Ref. [16].

The paper is organized as follow: in Sec. II we introduce our model and discuss its main properties. In Sec. III we derive the dynamical equations for soliton collective coordinates and obtain an expression of the average kink velocity as a function of the system parameters. In Sec. IV we compare our analytical results with direct numerical simulations and discuss them in connection with previous work. Finally, in Sec. V the main conclusions of the paper are summarized.

\section{THE MODEL}

Let us consider the following perturbed asymmetric double sine-Gordon equation

$$
\begin{aligned}
\phi_{t t}-\phi_{x x}+\sin (\phi)+\lambda \cos (2 \phi) & =F\left(x, t, \phi, \phi_{t}, \ldots\right) \\
& \equiv f(t)-\alpha \phi_{t},
\end{aligned}
$$

where $\lambda \in[-1,1]$ is a parameter related to the asymmetry of the nonlinear Klein-Gordon potential, $\alpha$ is a damping constant, and $f(t)=\epsilon \sin \left(\omega t+\theta_{0}\right)$ is a periodic force with amplitude $\epsilon$, frequency $\omega$, and phase $\theta_{0}$. This system is connected with interesting physical problems, such as arrays of inductively coupled asymmetric superconducting quantum interference devices of the type considered in Ref. [21]. A mechanical analog of Eq. (1) in terms of a chain of a double pendulum was given in Ref. [22]. For $F=0$, Eq. (1) has a Hamiltonian structure with Hamiltonian (energy)

$$
H=\int_{-\infty}^{+\infty} d x\left\{\frac{1}{2}\left(\phi_{t}^{2}+\phi_{x}^{2}\right)+U(\phi)\right\},
$$

and momentum

$$
P=-\int_{-\infty}^{+\infty} d x \phi_{x} \phi_{t}
$$

The potential in Eq. (2) is $U(\phi)=C-\cos (\phi)+(\lambda / 2) \sin (2 \phi)$, $C=\cos \left(\phi_{0}\right)-(\lambda / 2) \sin \left(2 \phi_{0}\right)$, and $\phi_{0}=\arcsin [(1-A) /(4 \lambda)]$ $+2 \pi n$ (notice that for $\lambda \neq 0$ the potential is asymmetric in the field variable). Similar to the sine-Gordon equation, the energy and the momentum are both conserved quantities for the unperturbed ADSGE. In this case it was shown [16] that Eq. (1) has an exact kink (antikink) solution of the form

$$
\phi_{K}^{ \pm}=\phi_{0}+2 \tan ^{-1}\left\{\frac{\operatorname{sign}(\lambda) A B}{A-1-\eta \sinh \left[ \pm \frac{\xi}{2} \sqrt{\frac{A B}{|\lambda|}}\right]}\right\},
$$

where $\xi=(x-V t) / \sqrt{1-V^{2}}, A=\sqrt{1+8 \lambda^{2}}, \quad \eta=2 \lambda \sqrt{2(1+A)}, \quad B$ $=\sqrt{2\left(4 \lambda^{2}-1+A\right)}$ (the \pm signs refer to the kink and antikink solutions, respectively). In the limit $\lambda \rightarrow 0$ (zero asymmetry),
Eq. (4) reduces to the well known soliton solution of sineGordon equation.

\section{COLLECTIVE COORDINATE ANALYSIS}

The term $F\left(x, t, \phi, \phi_{t}\right)$ added to the ADSGE can be considered as a small perturbation acting on the system. In this case the energy and the momentum will depend on time, so that it is natural to assume an ansatz for the perturbed kink of the form

$$
\phi=\phi_{0}+2 \tan ^{-1}\left\{\frac{\operatorname{sign}(\lambda) A B}{A-1-\eta \sinh \left[\frac{x-X(t)}{W(t)}\right]}\right\},
$$

where $X(t)$ and $W(t)$ represent dynamical collective coordinates corresponding to the center of mass and the width of the kink, respectively (a similar approach was introduced in [23] for the SGE). In the following, we consider only kink solutions since the analysis of antikinks will follow from it without difficulty. In absence of perturbation, the kink moves with constant velocity $V$ and constant width $W(t)=W_{s}$ $=W_{0} \sqrt{1-V^{2}}\left(W_{0}=2 \sqrt{|\lambda| /[A B]}\right)$, so that $X(t)=V t$. The kink momentum calculated from Eq. (3) is given by $P$ $=M(\lambda) V / \sqrt{1-V^{2}}$ with $M(\lambda)=\frac{1}{2} R^{2} I_{1}(\lambda) \sqrt{A B /|\lambda|}$, with $R$ and $I_{1}(\lambda)$ defined below. Notice that in the ansatz (5) we do not assume any relativistic relation between the velocity and the momentum of the kink since the relativistic invariance of the theory is automatically broken by the perturbation. This implies that the velocity of the kink should not be obtained from the relativistic expression of the momentum but should be computed directly from the time derivative of the center of mass of the kink. We also remark that ansatz (5) is consistent with the linear stability analysis performed in Ref. [16], showing the existence of an internal mode frequency $\Omega_{I}$ below the phonon band (this mode disappears in the sineGordon limit $\lambda=0$ ). From the spatial profile of the corresponding localized eigenfunction, indeed, one can see that the internal mode is linked to vibrations of the kink's width, this suggesting the choice of ansatz (5). We remark that a more general ansatz including the phonon's dressing [24] requires one to solve the Sturm-Liouville problem corresponding to the linearized ADSGE around the kink solution (4), this being a very difficult task to solve analytically (for a numerical solution of this problem, see [16]).

By substituting Eq. (5) into Eqs. (2) and (3), and differentiating with respect to time, one obtains, after straightforward calculations (for details, see [25]), that $X(t)$ and $W(t)$ satisfy the following system of nonlinear ODE:

$$
\begin{gathered}
\frac{d X}{d t}=\frac{P(t) W(t)}{R^{2} I_{1}}-\frac{I_{2}}{I_{1}} \dot{W} \\
\dot{W}^{2}-2 W \ddot{W}-2 \alpha W \dot{W}=-\frac{I_{1}}{K}+\frac{W^{2}}{K}\left[\frac{P^{2}}{R^{4} I_{1}}+\left(\operatorname{sign}(\lambda) I_{4}\right.\right. \\
\left.\left.-q \frac{I_{2}}{R I_{1}}\right) \frac{2 f(t)}{R}+\frac{2}{R^{2}} g\right],
\end{gathered}
$$

where the momentum $P$ is a solution of the equation 


$$
\frac{d P}{d t}=-\alpha P-q f(t),
$$

with $q=2 \pi$ and

$$
\begin{gathered}
I_{i}=\int_{-\infty}^{+\infty} \frac{\cosh ^{2}(\theta) \theta^{i-1} d \theta}{\left[(A-1-\eta \sinh [\theta])^{2}+A^{2} B^{2}\right]^{2}}, \quad i=1,2,3, \\
I_{4}=\int_{-\infty}^{+\infty} \frac{\cosh (\theta) \theta d \theta}{(A-1-\eta \sinh [\theta])^{2}+A^{2} B^{2}}, \\
K=\left(I_{3}-\frac{I_{2}^{2}}{I_{1}}\right), \quad R=2 A B \eta, \quad g=\frac{I_{1}(\lambda) A^{3} B^{3} \eta^{2}}{2|\lambda|} .
\end{gathered}
$$

Notice that the above integrals depend on the asymmetry parameter $\lambda$ and are finite for any value of $\lambda$ (they can be easily calculated by numerical tools). In Ref. [19] a similar approach was used to show that the internal mode mechanism gives rise to net motion in the $\mathrm{SG}$ equation perturbed by two harmonic forces. However, the present mechanism of soliton ratchets is slightly different from the one considered in Ref. [19] since in our case the symmetric external force acts directly on the translational mode and on the kink's width oscillations (internal mode), the resonance of these two degrees of freedom makes possible, in presence of the ldamping, the net motion.

In order to solve the system of equations $(6 a),(6 b)$, and (7), we use the following initial conditions: $d X(0) / d t=0$, $P(t=0)=P(0)$, and $W(0)=W_{0}$. We solve first the noncoupled linear equation for the total momentum. From Eq. (7) we obtain

$$
\begin{aligned}
P(t)= & \frac{-q \epsilon}{\sqrt{\alpha^{2}+\omega^{2}}} \sin \left[\omega t+\theta_{0}+\delta\right] \\
& +\mathrm{e}^{-\alpha t}\left[P(0)+\frac{q \epsilon}{\sqrt{\alpha^{2}+\omega^{2}}} \sin \left(\theta_{0}+\delta\right)\right],
\end{aligned}
$$

where $\tan (\delta)=-\omega / \alpha$ and the last term in the right-hand side, being a transient, will be neglected (we are interested in the stationary regime $t \gg \alpha^{-1}$ ). We remark that in the stationary regime, $P(t)$ is a sinusoidal function of time and, therefore, its time-average $\langle P(t)\rangle$ is zero notice that, as remarked in [26], Eq. (7) is an exact evolution equation for the momentum (3) valid for any periodic potential $U(\phi)$ and any external ac force $f(t)\}$. The fact that the time-average of the momentum is zero, however, does not mean that there is not transport because as previously remarked, the drift velocity $d X / d t$ is not linked to the momentum by the usual relativistic relation. From Eq. (6b) we see that the width of the kink is affected directly by the ac force with a frequency $\omega$ and indirectly by the translational motion (the momentum) with a frequency $2 \omega$ (see Ref. [25(b)]). Since it is difficult to solve exactly this equation, we will search for an approximate solution for $W(t)$ in the form of a power series in $\epsilon$ :

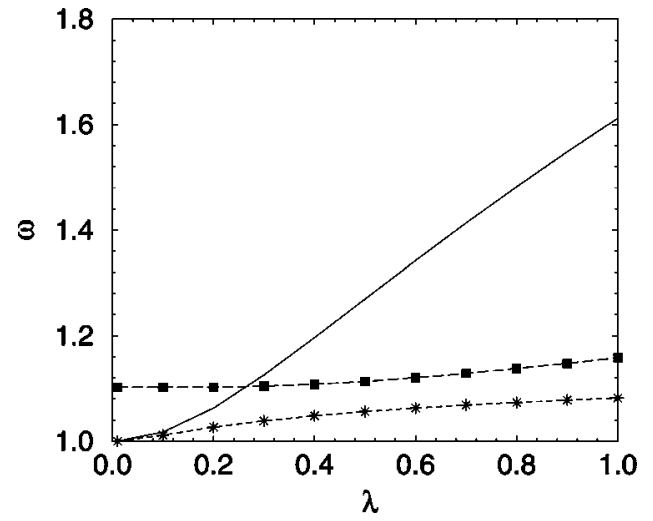

FIG. 1. Small oscillation spectrum of the ADSG and the frequency $\Omega$ (black squares) of the kink's width oscillations, as a function of $\lambda$. The continuous curve denotes the lower edge of the phonon band, while the stars joined by the dashed lines refer to the internal mode frequency $\Omega_{I}$.

$$
W(t)=W_{0}+\epsilon W_{1}(t)+\epsilon^{2} W_{2}(t)+O\left(\epsilon^{3}\right) .
$$

Inserting (9) into (6b) and taking terms of the same order in $\epsilon$, we obtain, after a transient time $\left(t \gg \alpha^{-1}\right)$, the following set of linear equations. At order $O(\epsilon)$ we have

$$
\ddot{W}_{1}+\Omega^{2} W_{1}+\alpha \ddot{W}_{1}=W_{0} G(\lambda) \sin \left(\omega t+\theta_{0}\right),
$$

where

$$
\begin{gathered}
\Omega^{2}=\frac{2 g(\lambda)}{R^{2} k(\lambda)}, \\
G(\lambda)=\frac{q I_{2}-\operatorname{sgn}(\lambda) I_{1} I_{4} R}{\left(I_{1} I_{3}-I_{2}^{2}\right) R^{2}},
\end{gathered}
$$

while at the second-order $\left[O\left(\epsilon^{2}\right)\right]$ we obtain

$$
\begin{aligned}
\ddot{W}_{2}+\Omega^{2} W_{2}+\alpha \dot{W}_{2}= & \frac{\dot{W}_{1}^{2}}{2 W_{0}}+\frac{\Omega^{2} W_{1}^{2}}{2 W_{0}}-\frac{W_{0} q^{2} \sin ^{2}\left[\omega t+\theta_{0}+\delta\right]}{2 R^{4}\left(I_{1} I_{3}-I_{2}^{2}\right)\left(\alpha^{2}+\omega^{2}\right)} \\
& +G(\lambda) W_{1} \sin \left(\omega t+\theta_{0}\right) .
\end{aligned}
$$

Equations (10) and (13) correspond to linear, damped, and driven oscillators with characteristic frequency $\Omega$. We find that for $\lambda \in[-1,1]$ the values of $\Omega$ are quite close to the internal mode frequency $\Omega_{I}$ of the ADSGE. This is shown in Fig. 1, where the small oscillation spectrum and the frequency $\Omega$ versus $\lambda$ are plotted. Notice that the maximum difference between $\Omega$ and $\Omega_{I}$ is $\approx 0.1$. It is also interesting to note that while for small values of $\lambda$ (i.e., close to the sineGordon limit), $\Omega$ lies in the phonon band, away from this limit; i.e., for $0.24<\lambda<1, \Omega$ is well below the phonon band and the lifetime of the $W(t)$ oscillation becomes infinite. The fact that the $W(t)$ oscillation become important in the same parameter region where the ratchet effect exists (large potential asymmetries) indicates the importance of this mode in describing the phenomenon. 
To obtain an analytical expression for the mean velocity of the kink, we take advantage of the fact that Eqs. (10) and (13) for the lowest-order terms of the $W(t)$ mode can be exactly solved.
The homogeneous part of the solutions of both equations decays to zero after a transient time, if $\alpha^{2}-4 \Omega^{2}<0$. The particular solutions of (10) and (13) are given, respectively, by

$$
\begin{gathered}
W_{1}(t)=-\frac{W_{0} G(\lambda) \cos \left(\omega t+\theta_{0}+\tilde{\theta}\right)}{\sqrt{\left(\Omega^{2}-\omega^{2}\right)^{2}+\alpha^{2} \omega^{2}}}, \\
W_{2}(t)=\frac{A_{0}}{\Omega^{2}\left[\left(\Omega^{2}-\omega^{2}\right)^{2}+\alpha^{2} \omega^{2}\right]}+\frac{A_{1} \sin \left(2 \omega t+2 \theta_{0}+2 \tilde{\theta}+\tilde{\theta}_{1}\right)}{\left[\left(\Omega^{2}-\omega^{2}\right)^{2}+\alpha^{2} \omega^{2}\right] \sqrt{\left(\Omega^{2}-4 \omega^{2}\right)^{2}+4 \alpha^{2} \omega^{2}}} \\
+\frac{A_{2} \sin \left(2 \omega t+2 \theta_{0}+2 \delta+\tilde{\theta}_{1}\right)+A_{3} \cos \left(2 \omega t+2 \theta_{0}+\tilde{\theta}+\tilde{\theta}_{1}\right)}{\left[\left(\Omega^{2}-\omega^{2}\right)^{2}+\alpha^{2} \omega^{2}\right] \sqrt{\left(\Omega^{2}-4 \omega^{2}\right)^{2}+4 \alpha^{2} \omega^{2}}}
\end{gathered}
$$

where $\tan (\tilde{\theta})=\left(\Omega^{2}-\omega^{2}\right) /(\alpha \omega), \tan \left(\tilde{\theta}_{1}\right)=\left(\Omega^{2}-4 \omega^{2}\right) /(2 \alpha \omega)$, and

$$
\begin{gathered}
A_{0}=A_{1}-A_{2}+\frac{W_{0} G^{2} \Omega^{2}}{2}, \\
A_{1}=W_{0} G^{2}(\lambda)\left\{\frac{\Omega^{2}}{4}-\frac{\omega^{2}}{4}\right\}, \\
A_{2}=\frac{W_{0} q^{2}\left[\left(\Omega^{2}-\omega^{2}\right)^{2}+\alpha^{2} \omega^{2}\right]}{4 R^{4}\left(I_{1} I_{3}-I_{2}^{2}\right)\left(\alpha^{2}+\omega^{2}\right)}, \\
A_{3}=\frac{W_{0} G^{2}(\lambda) \sqrt{\left(\Omega^{2}-\omega^{2}\right)^{2}+\omega^{2} \alpha^{2}}}{2} .
\end{gathered}
$$

Substituting Eqs. (8) and (9) into Eq. (6a), we obtain that the velocity of the kink up to the second order in $\epsilon$ is given by

$$
\begin{aligned}
\frac{d X}{d t}= & \epsilon\left[\frac{-q W_{0} \sin \left(\omega t+\theta_{0}+\delta\right)}{R^{2} I_{1}(\lambda) \sqrt{\alpha^{2}+\omega^{2}}}-\frac{I_{2}(\lambda)}{I_{1}(\lambda)} \dot{W}_{1}\right] \\
& +\epsilon^{2}\left[\frac{-q W_{1} \sin \left(\omega t+\theta_{0}+\delta\right)}{R^{2} I_{1}(\lambda) \sqrt{\alpha^{2}+\omega^{2}}}-\frac{I_{2}(\lambda)}{I_{1}(\lambda)} \dot{W}_{2}\right] .
\end{aligned}
$$

By taking the average value of this velocity over one period $(T=2 \pi / \omega),\langle d X / d t\rangle \equiv\langle V\rangle=(1 / T) \int_{0}^{T}(d X / d t) d \tau$, we finally obtain

$$
\langle V\rangle=\frac{-\epsilon^{2} q W_{0} G(\lambda) \Omega^{2} \alpha}{2 R^{2} I_{1}(\lambda)\left(\alpha^{2}+\omega^{2}\right)\left[\left(\Omega^{2}-\omega^{2}\right)^{2}+\alpha^{2} \omega^{2}\right]} .
$$

Equations (16) and (17) represent the main result of the paper. From their analysis, the following important conclusions can be made. First, we notice that the nonzero average velocity is due to the effective interaction between the translational $[P(t)]$ and internal mode $\left[W_{1}(t)\right]$ represented by the first term in the second bracket of (16). Second, Eq. (17) shows that the average velocity does not depend on the ini- tial phase. Indeed, the direction of the motion is determined only by $G(\lambda)$ (i.e., by the parameter $\lambda$ ), which controls the asymmetry of the potential. It is not difficult to check that $G(\lambda)>0(G(\lambda)<0)$ for $\lambda>0(\lambda<0)$ and that $G(\lambda)$ vanishes at $\lambda=0$. Third, from Eq. (17) we also see that for a given frequency there is an optimal value of the damping to achieve the maximal mean velocity (see Fig. 4 of [16]). This optimal value can be easily calculated as a function of $\omega$ and $\lambda$ (see the solid line of Fig. 4 of the present paper), and is given by

$$
\alpha_{o p t}=\sqrt{\frac{-D-\omega^{4}+\sqrt{\left(D+\omega^{4}\right)^{2}+12 D \omega^{4}}}{6 \omega^{2}}},
$$

where $D=\left(\Omega^{2}-\omega^{2}\right)^{2}$.

Fourth, the activation of the internal mode alone, without any phonons present in the system, does not allow the damping to rectify the motion. By varying $\alpha$ in Eq. (17) we cannot change the sign of the average velocity. This feature confirms the prediction of [16], where the rectification of the movement for small damping and large amplitude of the ac force was related to the excitation of phonons for a given choice of parameters.

Moreover, the existence of a resonant behavior of the mean velocity as a function of the frequency $\omega$ is also qualitatively confirmed by Eq. (17). As reported in Ref. [16] (see Fig. 6 of this paper), $\langle V\rangle$ becomes maximum when $\omega$ approaches the internal mode frequency $\Omega_{I} \approx \Omega$. This agreement, however, is only qualitative since the resonant peak is very close to the edge of the phonon band, so that phonons are easily excited in the system. In fact, Eq. (14) together with Eq. (15) show that the kink oscillates with two frequencies $\omega$ and $2 \omega$. Close to the resonance $2 \omega \approx 2 \Omega$ one is inside the phonon band, so that phonons become excited and the $\mathrm{CC}$ analysis becomes unaccurate.

Notice that Eq. (17) is valid for small $\epsilon$ and for times ( $t$ $\left.\gg \alpha^{-1}\right)$; thus, for the zero-damping case, nothing can be inferred about the mean velocity. It is of interest to investigate 
the zero-damping case separately to check the role played by the damping in the phenomenon. For $\alpha=0$ the momentum equation (7) simplifies as well as, the collective coordinate equations (6a) and (6b). From Eq. (7) (with $\alpha=0$ ) we have that a solution satisfying the initial condition $P(0)=0$ is readily obtained as

$$
P(t)=\frac{q \epsilon}{\omega}\left[\cos \left(\omega t+\theta_{0}\right)-\cos \left(\theta_{0}\right)\right] .
$$

By substituting this equation and Eq. (9) into Eq. (6a), we obtain that the kink velocity at order $\epsilon$ is

$$
\frac{d X}{d t}=\frac{q W_{0} \epsilon}{R^{2} \omega I_{1}(\lambda)}\left[\cos \left(\omega t+\theta_{0}\right)-\cos \theta_{0}\right]-\frac{I_{2}(\lambda)}{I_{1}(\lambda)} \epsilon \dot{W}_{1} .
$$

The first-order correction to the soliton width $W_{1}$ can be calculated by solving Eq. (10) with $\alpha=0$. For $\omega \ll \Omega$ we obtain

$$
\begin{aligned}
W_{1}(t)= & -\frac{W_{0} G(\lambda) \cos \theta_{0} \sqrt{\omega^{2}+\Omega^{2} \tan ^{2} \theta_{0}}}{\Omega\left(\Omega^{2}-\omega^{2}\right)} \sin (\Omega t+\varphi) \\
& +\frac{W_{0} G(\lambda)}{\Omega^{2}-\omega^{2}} \sin \left(\omega t+\theta_{0}\right),
\end{aligned}
$$

where $\tan \varphi=(\Omega / \omega) \tan \theta_{0}$ in order to fulfill the initial conditions $W_{1}(0)=0$ and $\dot{W}_{1}(0)=0$. It is clear that the velocity of the kink depends on the initial phase $\theta_{0}$, so that, by averaging over the phase, one obtains zero transport. A straightforward calculation shows that the same result is also true at order $\epsilon^{2}$. This suggests that the ratchet effect in the zero-damping case does not exist. As we will see in the next section, this conclusion is confirmed by numerical simulations of Eq. (1).

\section{NUMERICAL SIMULATIONS AND DISCUSSION}

The CC analysis of the previous section neglects the presence of phonons in the system and is based on a particular ansatz for the soliton shape in Eq. (5). Moreover, the approximated solution (17) is valid only when the perturbations are small enough $(\epsilon \ll 1)$. To check our results, we compare numerical solutions of Eqs. (6a) and (6b) and the approximated solution in Eq. (17), with direct numerical integrations of Eq. (1). Numerical simulations of (1) were performed by using a fourth-order Runge-Kutta scheme [27] with time and space steps $\Delta t=0.01, \Delta x=0.1$, in the finite-length domain $x$ $\in[-50,50]$, taking into account 200 time periods. In order to obtain the numerical solutions of the $\mathrm{CC}$ equations, we have integrated Eqs. (6a) and (6b) with the routine DIVPRK of the IMSL library [28], which uses the Runge-Kutta-Verner sixthorder method.

Figures 2 and 3 show the mean velocity dependence on the damping coefficient for different values of the amplitude and frequency of the ac force. From Fig. 2 we see that for low values of $\epsilon$ there is a very good agreement between the $\mathrm{CC}$ results and PDE simulations in the whole range of $\alpha$. However, when the amplitude of the ac force is increased, the approximated solution (17) deviates from the numerical

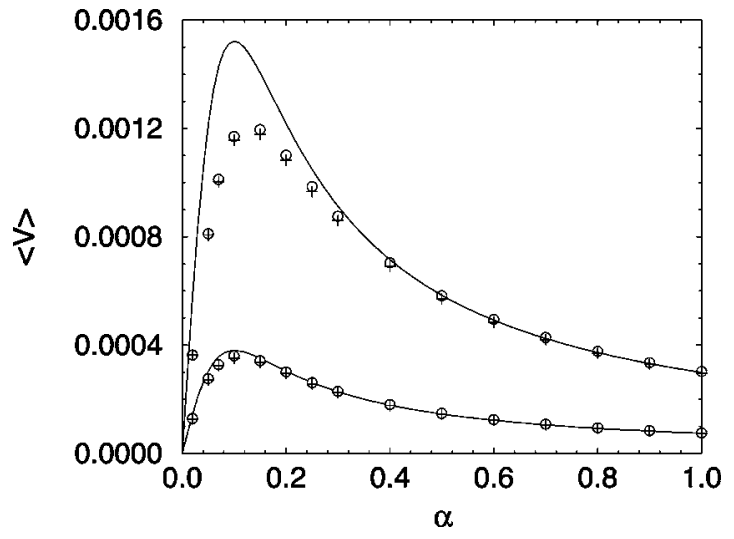

FIG. 2. Dependence of the mean kink velocity on the damping coefficient for two different values of strength of the ac force $\epsilon$ $=0.1$ (upper curves) and $\epsilon=0.05$ (lower curves). Other parameters are fixed as $\lambda=-0.5, \omega=0.1$, and $\theta_{0}=\pi / 2$. The solid lines represent the approximate solution of the CC theory [Eq. (17)], whereas circles and pluses refer, respectively, to PDE numerical simulations of (1) and numerical solutions of the CC equations (6a) and (6b).

solution of the $\mathrm{CC}$ equations and from the PDE simulations, mostly in the optimal damping region. In Fig. 3 we show the same dependence for a higher value of the driver frequency $(\omega=0.4)$. We see that, although the approximated solution (17) and the numerical solution of the CC equations are quite close, there is a discrepancy with the PDE results starting from the peak and extending to higher values of $\alpha$, this indicating that the CC ansatz is valid only for $\omega \ll \omega_{p h}\left(\omega_{p h}\right.$ $=1.2699$ for $\lambda=-0.5$ ).

In Fig. 4, we check Eq. (18) for the optimal value $\alpha_{o p t}(\omega)$ of the damping constant for $\lambda=-0.5$. We can see that this value increases up to $\omega \approx 0.7$ and after that $\alpha_{\text {opt }}(\omega)$ decreases. Again, a good agreement between the CC theory and PDE results is found at small $\omega$ values.

By increasing the amplitude $\epsilon$ of the driver, reversal of current can also occur [16]. This is shown in Fig. 5, from which we see that as $\alpha$ is increased, the mean velocity com-

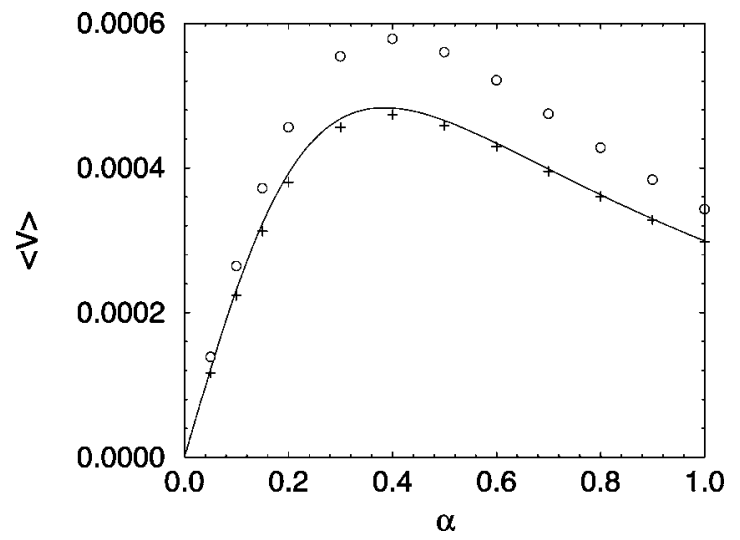

FIG. 3. Mean kink velocity versus the damping coefficient. Parameters are $\lambda=-0.5, \epsilon=0.1, \omega=0.4$, and $\theta_{0}=\pi / 2$. The solid line represents the approximated solution of the CC theory [Eq. (17)], whereas circles and pluses are the results of the numerical simulations of the PDE (1) and the numerical solutions of the CC equations (6a) and (6b), respectively. 


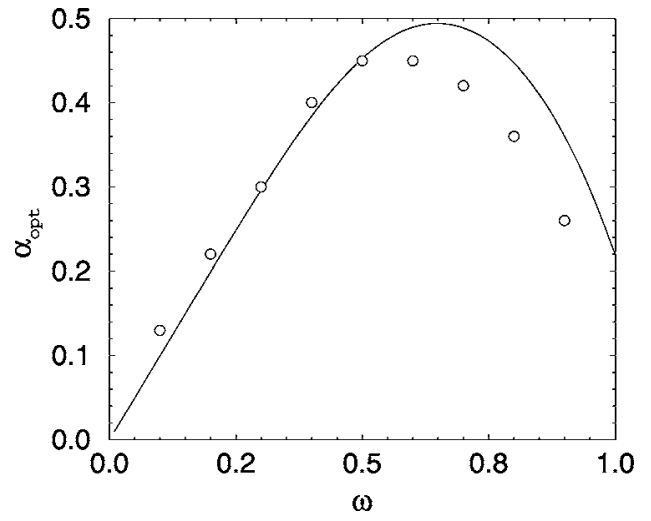

FIG. 4. Optimal value of the damping as a function of the frequency of the ac force for $\lambda=-0.5$. The solid line represents the results obtained from Eq. (18) while the circles refer to numerical simulations of Eq. (1).

puted from PDE simulations displays a crossover from negative to positive values (i.e., a current reversal occurred). This phenomenon is described neither by the $\mathrm{CC}$ analysis (notice that Eq. (17) predicts a positive average velocity for all $\lambda$ $<0$ ), nor by the numerical solutions of the CC analysis depicted in the figure. This agrees with the claim made in Ref. [16] that reversal currents do not depend on the internal mode mechanism, but rather on the existence of phonons in the system. In order to show the presence of phonon modes when this phenomenon appears, we have plotted in Fig. 6 the discrete Fourier transform (DFT) of the kink's width $W(t)$ (obtained from the numerical simulations of the PDE) for two values of $\alpha$ : $\alpha=0.2$ (before crossover occurs) and $\alpha$ $=0.7$ (inside the rectified motion region). In the former case (upper panel), one of the frequencies of the oscillations of $W(t)$ lies inside the phonon band, so that phonons are clearly excited. In the latter case (lower panel) the main frequencies of the spectrum are well below the lower phonon edge $\left(w_{p h} /(2 \pi) \approx 0.2\right)$. From this we conclude that phonon modes

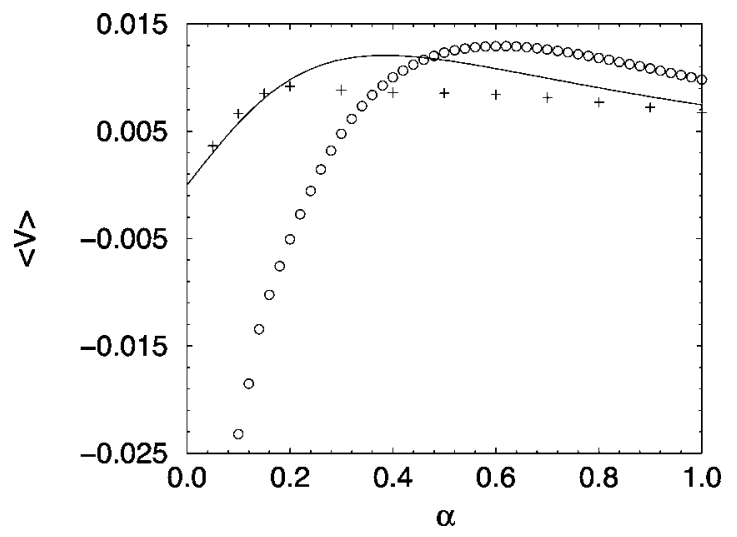

FIG. 5. Mean kink velocity versus the damping coefficient for $\lambda=-0.5, \epsilon=0.5, \omega=0.4$, and $\theta_{0}=\pi / 2$. The solid line represents the approximated solution of the CC theory [Eq. (17)], while circles and pluses denote the results of the numerical simulations of the PDE (1) and of the numerical solutions of the CC equations (6a) and (6b), respectively. The PDE results show that for very small values of damping $(\alpha<0.1)$, pairs of kink and antikink appear.
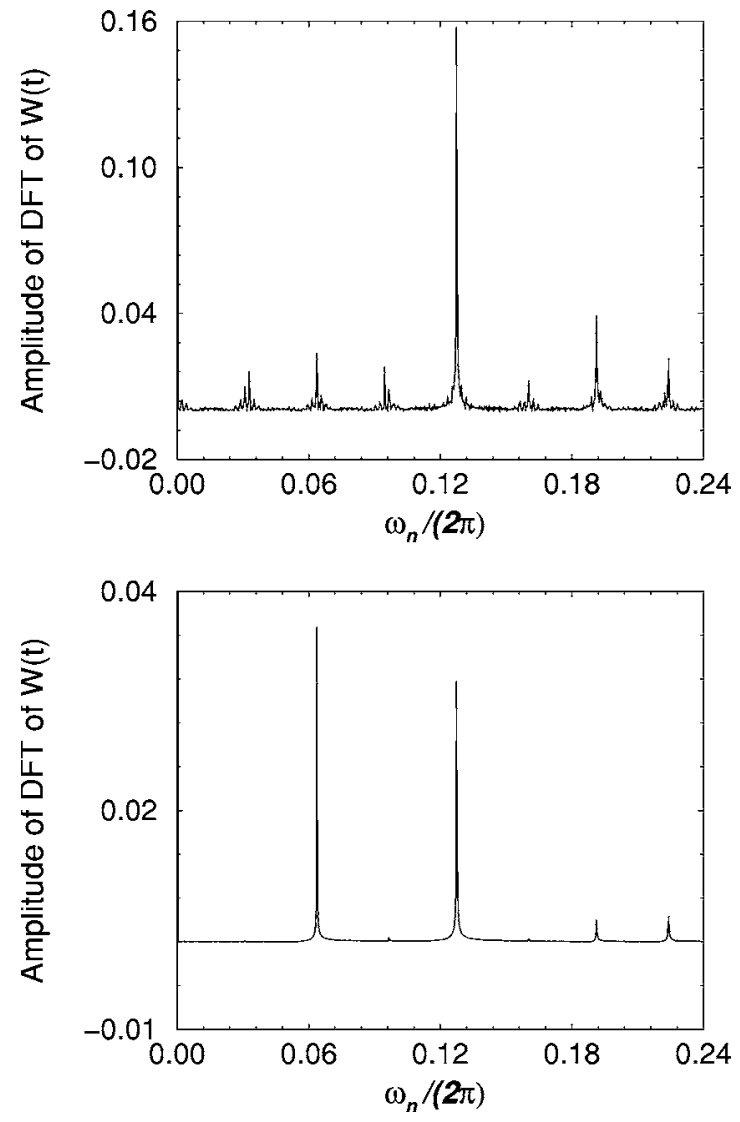

FIG. 6. DFT of the width of the kink $[W(t)]$ obtained from the numerical solutions of the PDE for the same parameters of Fig. 5 and $\alpha=0.1$ (upper panel) and $\alpha=0.7$ (lower panel, when the current is rectified). The upper panel refers to the case in which a current reversal occurs. The relevant frequencies in the spectrum are: $\omega_{1}$ $=2 \omega=0.8 ; \omega_{2}=3 \omega=1.2 ; \omega_{3}=\omega=0.4 ;$ and $\omega_{4}=(7 / 2) \omega=1.4$ (this last being inside the phonon band). The lower panel corresponds to the case in which the current is rectified. The main frequencies in this case are located at $\omega_{1}=\omega=0.4$ and $\omega_{2}=2 \omega=0.8$ away from the phonon band. The frequency of the internal mode and the lower phonon's frequency are $\Omega_{I}=1.056$ and $\omega_{p h}=1.269$, respectively.

are important for current reversals and a theory based on the internal mode alone (as the one presented here) cannot describe properly this phenomenon.

In Ref. [16] a resonant behavior of mean velocity as a function of $\omega$ was also reported (see the peak of $\langle V\rangle$ at $\omega$ $\approx \Omega_{I}$ in Fig. 3 of this paper). This feature is also confirmed by Eq. (17) (notice that the denominator of this expression has a minimum at $\omega=\Omega \approx \Omega_{I}$ ), although, in this case, the agreement with PDE results is only qualitative. This is shown in Fig. 7, from which we see the presence of a resonant structure, with good agreement at small frequencies and large deviations from PDE results at higher $(\omega>0.6)$ values. The agreement at low frequencies can be understood from the fact that phonons in this case are hardly excited and the $\mathrm{CC}$ description becomes accurate. On the contrary, when $\omega$ gets close to the resonant peak, phonons are easily excited and the $\mathrm{CC}$ analysis becomes unadequate.

We have also investigated the dependence of the phenomenon on the asymmetry parameter $\lambda$ as well as the impor- 


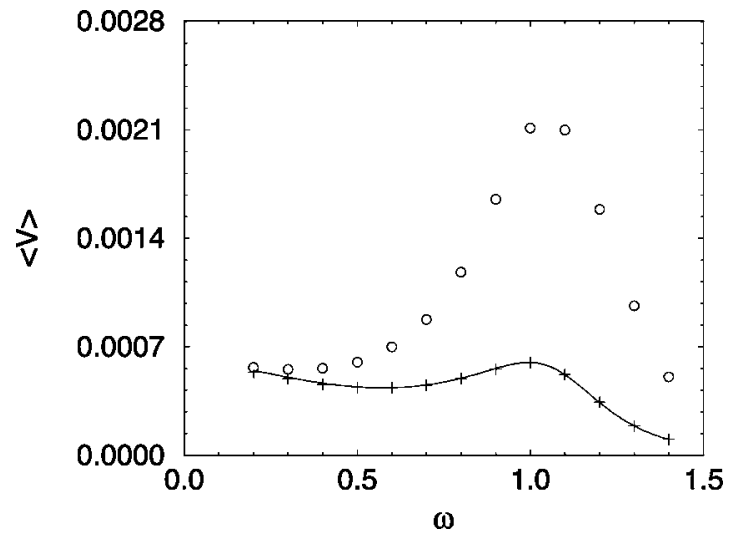

FIG. 7. Mean velocity of the kink as a function of $\omega$. The pluses superimposing the solid line show a good agreement between the approximated (solid line) and the numerical (pluses) solutions of the CC theory. Results obtained from the integration of the Eq. (1) (circles) coincide with the CC theory only for smaller values of $\omega$. Parameters are fixed as $\lambda=-0.5, \epsilon=0.1, \theta_{0}=\pi / 2$, and $\alpha=0.5$.

tance of the damping term for soliton ratchets. In Fig. 8 we show the mean velocity as a function of $\lambda$ for fixed system parameters and two different values of $\alpha$. We see that the curve is antisymmetric around the origin meaning that the sign of $\lambda$ determines the direction of motion, the maximal effect occurring around $|\lambda|=0.5$; i.e., the point of maximal asymmetry of the potential.

Finally, we have investigated the zero-damping limit of the phenomenon. In Fig. 9 we depict the time evolution of the center of mass of the kink for $\alpha=0$ and for different values of the initial condition phase $\theta_{0}$ of the ac force. We see that $X(t)$ is basically a linear function of time, whose slope depends on $\cos \left(\theta_{0}\right)$. This implies that, in this case, it is the initial phase that determines the direction of the motion and not only the asymmetry of the potential, as predicted by the $\mathrm{CC}$ analysis. Since in most experimental contexts the initial phase is usually unknown, one must consider the phase as a random variable and take an average of it. This

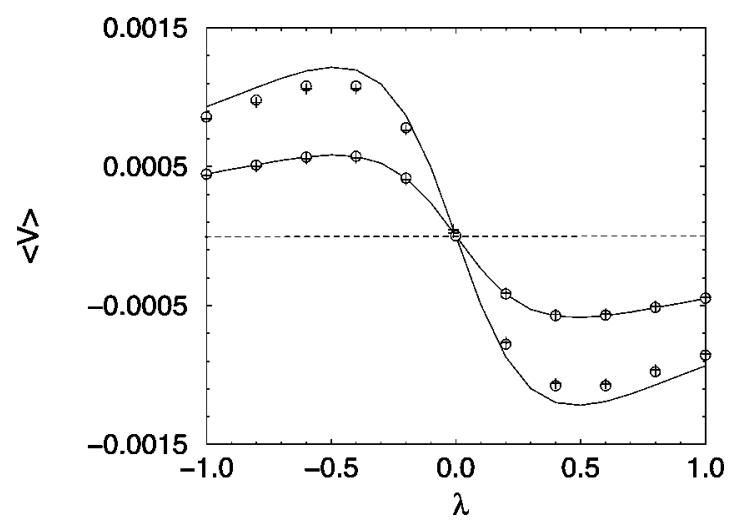

FIG. 8. Mean velocity of the kink center of mass versus $\lambda$ for $\epsilon=0.1, \omega=0.1, \theta_{0}=\pi / 2$, and for two values of the damping coefficient: $\alpha=0.2$ and $\alpha=0.5$ (for fixed $\lambda$, the mean velocity of the $\alpha$ $=0.5$ case is smaller).

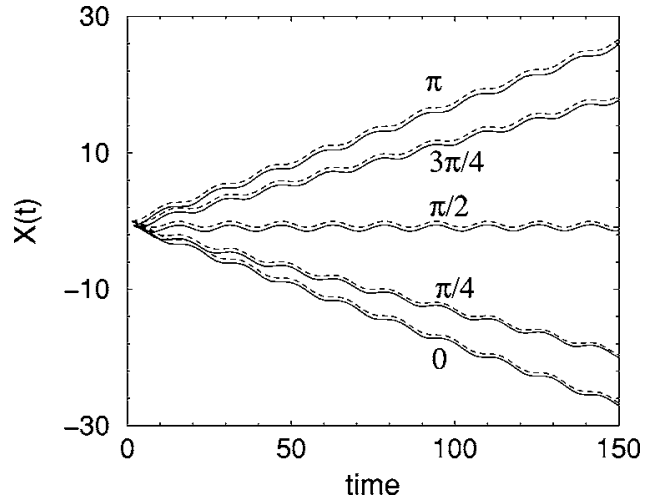

FIG. 9. Time evolution of the center of the kink for different values of initial phase, $\theta_{0}$, in the nondamped case. Parameters are fixed as: $\lambda=-0.5, \epsilon=0.1$, and $\omega=0.4$. The solid and the dashed lines represent the numerical simulations of Eq. (1) and the numerical solutions of Eqs. (6a) and (6b), respectively.

obviously implies that soliton ratchets cannot exist in the zero-damping limit.

\section{CONCLUSIONS}

In this paper we have studied the ratchet dynamics of the kink solution of the ADSGE by using a collective coordinate approach with two collective variables: the center of mass and the width of the kink. For these variables we have obtained a system of ODEs from which we derived an approximated expression for the mean velocity of the kink as a function of the system parameters. We have confirmed that the $\mathrm{CC}$ approach is valid when phonons are not excited in the system; i.e., for small values of $\epsilon$ and for $\omega \ll \omega_{p h}$. We have shown that for a proper description of soliton ratchets it is not enough to consider the kink as a point particle moving in a ratchet potential $[11,29]$ but it is crucial to include also the internal oscillations of the kink. In particular, we have shown that the net motion of the kink becomes possible due to the coupling between the translational mode and the internal coordinate with the ac force (the effective coupling being possible only in presence of damping when the time-average velocity becomes independent of the initial phase of the driver). We also showed that the asymmetry of the potential determines the direction of the motion and that in the zerodamping case the ratchet effect vanishes (i.e., it depends on initial conditions). The resonant behavior of the velocity as a function of frequency and damping was also investigated. We found that the mean velocity approach a maximum value when the frequency of the ac force goes to the internal mode frequency or when the damping coefficient approach its optimal value. Finally, we have shown that the occurrence of current reversal is related to the presence of phonons in the system rather than the coupling between the translational and the internal mode.

In conclusion, the results of our analysis confirm the internal mode mechanism for soliton ratchet proposed in Ref. [16] and provide an approximate analytical description of the phenomenon. 


\section{ACKNOWLEDGMENTS}

N.R.Q. acknowledges financial support from the Ministerio de Ciencia y Tecnología of Spain under the Grant No.
BFM2001-3878-C02, and from the Junta de Andalucía through the project FQM-0207. M.S. acknowledges financial support from the MURST, under a PRIN-2003 Initiative.
[1] M. O. Magnasco, Phys. Rev. Lett. 71, 1477 (1993); C. R. Doering, Nuovo Cimento Soc. Ital. Fis., D 17, 685 (1995).

[2] P. Hänggi and R. Bartussek, Nonlinear Physics of Complex System-Current Status and Future Trends, Lecture notes in Physics Vol. 746, J. Parisi et al. (Springer, Berlin, 1996), p. 476.

[3] S. Cilla, F. Falo, and L. M. Floría, Phys. Rev. E 63, 031110 (2001).

[4] P. Reimann and P. Häggi, Appl. Phys. A: Mater. Sci. Process. 75, 169 (2002).

[5] F. Marchesoni, Phys. Rev. Lett. 77, 2364 (1996).

[6] A. V. Savin, G. P. Tsironis, and A. V. Zolotaryuk, Phys. Lett. A 229, 279 (1997); Phys. Rev. E 56, 2457 (1997).

[7] F. Falo, P. J. Martínez, J. J. Mazo, and S. Cilla, Europhys. Lett. 45, 700 (1999); E. Trías, J. J. Mazo, F. Falo, and T. P. Orlando, Phys. Rev. E 61, 2257 (2000).

[8] L. Morales-Molina, F. G. Mertens, and A. Sánchez, Eur. Phys. J. B 37, 79 (2004).

[9] L. P. Faucheux, L. S. Bourdieu, P. D. Kaplan, and A. J. Libchaber, Phys. Rev. Lett. 74, 1504 (1995).

[10] C. S. Lee, B. Jankó, I. Derényi, and A. L. Barabási, Nature (London) 440, 337 (1999).

[11] G. Carapella and G. Costabile, Phys. Rev. Lett. 87, 077002 (2001).

[12] A. V. Ustinov, C. Coqui, A. Kemp, Y. Zolotaryuk, and M. Salerno, Phys. Rev. Lett. 93, 087001 (2004).

[13] P. Reimann, Phys. Rep. 361, 57 (2002).

[14] R. Bartussek, P. Hänggi, and J. G. Kissner, Europhys. Lett. 28, 459 (1994).

[15] P. Reimann, R. Bartussek, R. Häussler, and P. Hänggi, Phys. Lett. A 215, 26 (1996).
[16] Mario Salerno and Niurka R. Quintero, Phys. Rev. E 65, 025602(R) (2002)

[17] Mario Salerno and Yaroslav Zolotaryuk, Phys. Rev. E 65, 056603 (2002).

[18] S. Flach, Y. Zolotaryuk, A. E. Miroshnichenko, and M. V. Fistul, Phys. Rev. Lett. 88, 184101 (2002).

[19] L. Morales-Molina, N. R. Quintero, F. G. Mertens, and A. Sánchez, Phys. Rev. Lett. 91, 234102 (2003).

[20] G. Costantini, F. Marchesoni, and M. Borromeo, Phys. Rev. E 65, 051103 (2002).

[21] I. Zapata, R. Bartussek, F. Sols, and P. Hänggi, Phys. Rev. Lett. 77, 2292 (1996).

[22] Mario Salerno, Physica D 17, 227 (1985).

[23] M. J. Rice and E. J. Mele, Solid State Commun. 35, 487 (1980); Mario Salerno and Alwyn C. Scott, Phys. Rev. B 26, 2474 (1982).

[24] C. R. Willis and M. Farzaneh, Phys. Rev. E 69, 056612 (2004).

[25] (a) F. G. Mertens, H. J. Schnitzer, and A. R. Bishop, Phys. Rev. B 56, 2510 (1997); (b) N. R. Quintero, A. Sánchez, and F. G. Mertens, Phys. Rev. E 62, 5695 (2000); (c) N. R. Quintero and Elías Zamora-Sillero, Physica D 197, 63 (2004).

[26] N. R. Quintero, Bernardo Sánchez-Rey, and Jesús CasadoPascual, Phys. Rev. E 71, 058601 (2005).

[27] W. H. Press, S. A. Teukolsky, W. T. Vetterling, and B. P. Flannery, Numerical Recipes in Fortran 2nd Edition (Cambridge University Press, Cambridge, UK, 1992).

[28] IMSL MATH/LIBRARY Special Functions (Visual Numerics, Inc., Houston, TX, 1997).

[29] E. Goldobin, A. Sterck, and D. Koelle, Phys. Rev. E 63, 031111 (2001). 